\title{
Flow Simulation Using Local Grid Refinements to Model Laminated Reservoirs
}

\author{
Manuel Gomes Correia ${ }^{*}$, Célio Maschio and Denis José Schiozer \\ CEPETRO/FEM - University of Campinas (UNICAMP), Postal Code 6052 13083-970, Campinas, São Paulo - Brasil \\ e-mail: manuel@dep.fem.unicamp.br \\ *Corresponding author
}

\begin{abstract}
Super-giant carbonate fields, such as Ghawar, in Saudi Arabia, and Lula, at the Brazilian pre-salt, show highly heterogeneous behavior that is linked to high permeability intervals in thin layers. This article applies Local Grid Refinements (LGR) integrated with upscaling procedures to improve the representation of highly laminated reservoirs in flow simulation by preserving the static properties and dynamic trends from geological model. This work was developed in five main steps: (1) define a conventional coarse grid, (2) define LGR in the conventional coarse grid according to super-k and well locations, (3) apply an upscaling procedure for all scenarios, (4) define LGR directly in the simulation model, without integrate geological trends in LGR and (5) compare the dynamic response for all cases. To check results and compare upscaling matches, was used the benchmark model UNISIM-II-R, a refined model based on a combination of Brazilian Pre-salt and Ghawar field information. The main results show that the upscaling of geological models for coarse grid with LGR in highly permeable thin layers provides a close dynamic representation of geological characterization compared to conventional coarse grid and LGR only near-wells. Pseudo-relative permeability curves should be considered for (a) conventional coarse grid or (b) LGR scenarios under dual-medium flow simulations as the upscaling of discrete fracture networks and dual-medium flow models presents several limitations. The conventional approach of LGR directly in simulation model, presents worse results than LGR integrated with upscaling procedures as the extrapolation of dynamic properties to the coarse block mismatch the dynamic behavior from geological characterization. This work suggests further improvements for results for upscaling procedures that mask the flow behavior in highly laminated reservoirs.
\end{abstract}

\section{INTRODUCTION}

Super-giant carbonate fields, such as the mature Ghawar field in Saudi Arabia and newer Brazilian pre-salt reservoir discoveries in the Tupi area, present a specific set of challenges, which have prompted novel techniques. New techniques can be applied in a proven mature field for unconventional resources (heavy oil, bitumen, stranded gas). New techniques designed for new geological concepts (lacustrine carbonate plays) can also be applied to other recent discoveries, such as Brazilian pre-salt discoveries (Garland et al., 2012). Both giant fields show highly heterogeneous behavior due to the high permeability intervals associated with thin layers and/or horizontal fracture patterns. The layered lacustrine facies in Tupi, such as coquinas, microbial buildups and travertine shrubs are the main source of the high flow rates (Boyd et al., 2015), while the dolomite Ghawar Arab D formation is characterized by fractures and highly permeable thin layers called "super-k" zones (Voelker et al., 2003; AlDhafeeri and Nasr-El-Din, 2007; Eltom et al., 2013).

Representing and simulating these features through geological and numerical modeling is challenging. The integration of geological characterization with numerical 
simulation is commonly referred to as "upscaling". Many upscaling methods use averaging, homogenization or the latest techniques that use pseudo-functions or flow-based techniques (numerical methods). Among these upscaling methods, numerical methods are generally considered to be more accurate for permeability upscaling (Noetinger and Zargar, 2004). However, numerical methods depend on boundary conditions, leading to a non-unique solution (Preux et al., 2016). Local Grid Refinements (LGR) solve this problem by refining grid blocks for specific regions without the use of globally refined grids to avoid excessive simulation time.

LGR are widely applied in structured grids using commercial simulation software such as CMG or ECLIPSE (Peng et al., 2017). Due to large changes in saturation and pressure within well zones and, to ensure equivalent transmissibility rates and well indexes between coarse and fine grid simulations, near-well refinements are common approaches for complex flow mediums (Ding, 2004). Current research focuses on the development of new algorithms that couple near-well refinements (Ding, 2011; Li et al., 2014; Kheriji et al., 2015) and sensitivity studies based on grid refinement effects (Panja et al., 2013; Correia et al., 2014). Both phases of research focus on a complex flow medium, commonly linked to fractured carbonate reservoirs, which comprise approximately half of global, proven reserves (Ahr, 2008). Near-well refinement approaches for unconventional resources, such as tight and shale gas reservoirs, have become increasingly important to simulate near-well hydraulic fracturing (Ding et al., 2014).

\section{Motivation}

However, LGR are mainly applied to near-well regions and rarely used for inter-well regions in highly laminated reservoirs. Furthermore, LGR are normally applied directly in the coarse simulation model and disregarded in geomodeling and upscaling steps for a proper geological representation in flow simulation (Ding, 2011; Panja et al., 2013; Ding et al., 2014). Assessing the viability of LGR in the representation and flow simulation of highly permeable thin layers also extends to challenges in gridding and upscaling. This is because highly laminated reservoirs have the highest coefficient of variability, based on permeability and geological variability, resulting in highly heterogeneous reservoirs (Corbett and Jensen, 1992). These geological features characterize the supergiant Ghawar field in Saudi Arabia and super-giant Brazilian pre-salt reservoirs. Furthermore, to the best of our knowledge this is the first work that integrates upscaling procedures with LGR in order to preserve static properties, such as porosity and permeability, and dynamic trends from geological model.
To compare results and upscaling matches, we use the benchmark model UNISIM-II-R (Correia et al., 2015) as the reference model, a fine-grid model based on a combination of Brazilian Pre-salt and Saudi Ghawar field information. We assume the reference model to be high fidelity. The conventional coarse grid has an average grid-block size representative of a simulation model.

\section{OBJECTIVE}

The purpose of this work is to accurately represent highly laminated and fractured reservoirs in flow simulations, through the integration of upscaling procedures in LGR in order to preserve static properties and dynamic trends from geological model.

For a detailed analysis of the benefits of LGR integrated with upscaling procedures in flow simulation, comparing with (1) conventional coarse grid and (2) LGR only in simulation model, we separated the studies based on different structural grid scenarios and flow models. The comparisons focus on the following:

- Compare results from the LGR approach using a coarse grid with block corners (a) adjusted and (b) mismatched from the reference model UNISIM-II-R.

- Compare results from different LGR combinations according to well position and super-k layers.

- Compare results from LGR for (a) single-medium and (b) dual-medium flow models. We consider a geological scenario without fractures (single-medium) to compare the benefits of LGR in super-k layers (matrix) and compare its advantages with the presence of fractures (dual-medium flow model).

- Compare results from LGR integrated with upscaling procedures with LGR integrated with simulation model (LGR SIM).

\section{METHODOLOGY}

The proposed methodology follows five main steps:

- define a conventional coarse grid with block corners (a) aligned and (b) mismatched with reference model,

- define LGR in conventional coarse grid according to super-k and well location,

- apply the upscaling procedure for all cases,

- define LGR directly in the simulation model, without integrate geological trends in LGR,

- compare the dynamic response from flow simulation for all cases against the reference model.

The following sections focus on each step in more detail. 


\subsection{Define conventional coarse grid}

To analyze the influence of, and isolate, structural grid issues, we compare the upscaling matching between a conventional coarse grid and LGR for two structural grids: (1) Case A, the block corners from the coarse grid do not match the reference model; and (2) Case B, the block corners from the coarse grid are orientated and match the reference model.

Case A represents the conventional approach and reflects the difficulty of generating a coarse model with block corners perfectly matching those of the geological model, using geostatistical software, especially for strati graphically complex cases (e.g.: faults and folds). Case B is the theoretical approach to isolate structural grid mismatches through comparing the upscaled coarse grid and LGR with the reference solution.

\subsection{Apply LGR to Conventional Coarse Grid}

Most commercial flow simulators (e.g.: ECLIPSE, IMEX, PUMA FLOW) allow the use of LGR, refining regions of interest by applying local grids within the coarse grid. Some geostatistical software works with unconstructed grids or hybrid grids, a mixture of structured and unstructured grids. However, commercial black-oil flow simulators work only with structured grids based on finite difference methods. For simplicity and wide range of application, this work uses only structured grids. LGR are made to the host cell, which is the coarse block from the conventional coarse grid. To apply LGR, host cells must be defined according to regions of interest (wells, faults, facies, etc.), and the number of horizontal and vertical divisions that must be set for the host cells.

\subsection{Apply the Upscaling Procedure}

The reference model is upscaled, using a conventional procedure, for the output conventional coarse grid. We use the same method for the conventional grid with LGR. Through the upscaling procedure, petrophysical properties are populated for each refined block in LGR. Therefore, host cells (coarse blocks with LGR) are defined by a set of values depending on the number of host cell divisions (refinements). Also, each refined block in LGR is defined by respective rock-fluid data. Although comparing upscaling methods is not the focus of this work, the proper upscaling procedure for highly heterogeneous reservoirs is applied to the reference model to better evaluate the advantages of LGR against conventional coarse grid.

\subsection{Apply LGR SIM}

In this step, we apply LGR directly in simulation model (conventional method) according to regions of interest (wells, faults, facies, etc.). In this scenario we disregard the upscaling procedure from geologic model to LGR. Therefore, each refined block has the same value as the host cell.

\subsection{Compare the Dynamic Response from Flow Simulation for all Cases}

In this step, we compare all cases with the reference model, based on the dynamic response from flow simulation, through the cumulative oil production and cumulative water production of the field.

\section{APPLICATION}

The application section focuses on the first four steps of the methodology. We first describe the case study, and then detail the application of LGR. Thirdly, we focus on the upscaling method. Finally, we focus on the application of LGR SIM.

As mentioned before, we use two groups of models. Case A is the group of models (reference, coarse without LGR, and coarse with LGR) whose block corners from reference model do not match with conventional coarse grid. Case B is the group of models whose block corners from reference model match with conventional coarse grid. We assume the reference model to be a high fidelity model due to the high resolution of grid.

\subsection{Reference Model Description for Case A}

We base our study on the benchmark UNISIM-II-R (Correia et al., 2015), a refined model based on a combination of Brazilian Pre-salt and Ghawar field information. It is characterized by three facies (grainstone, packstone and super-k), and a small portion of non-reservoir layers. UNISIM-II-R grid cells measure $50 \times 50 \times 2 \mathrm{~m}$. For Case A, the conventional coarse grid block corners do not match those from UNISIM-II-R.

\subsection{Reference Model Description for Case B}

To match the grid block corners between reference model and coarse grid, the Case $\mathrm{B}$ was defined through a refinement of the conventional coarse grid from Case A. Therefore, the difference between grid block corners from Case A and Case $\mathrm{B}$, is the structural grid from the reference. The reference model is upscaled from refined model A using upscaling techniques appropriate for closing block volumes. For both cases, the structural grid is the same for the conventional coarse grid and LGR scenarios. Both refined models (A and B) have a similar block sizes. 


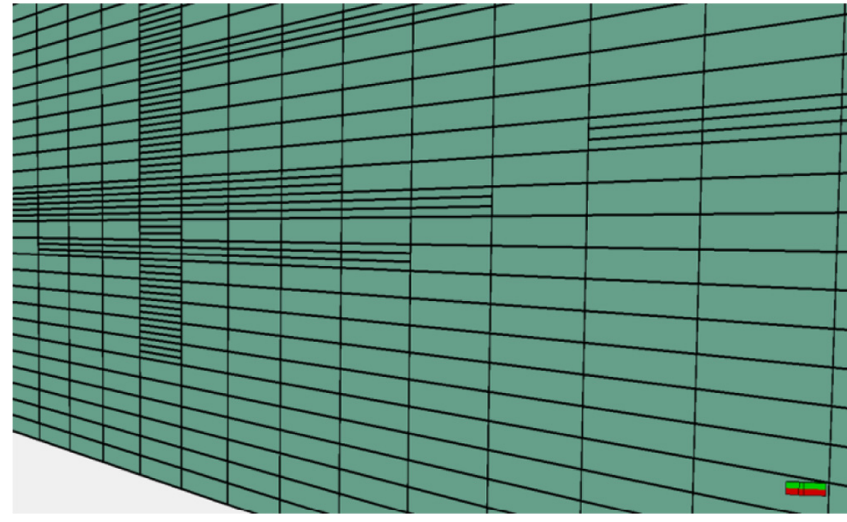

Figure 1

Grid for LGR scenario (super-k and well).

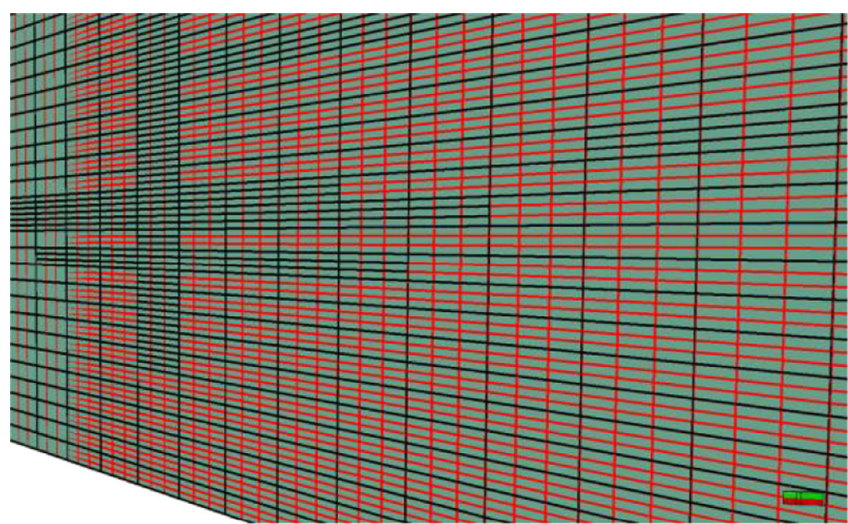

Figure 2

Grid for the reference model B overlapping the grid for LGR scenario.

Figure 1 shows the grid for LGR (super-k and well). Figure 2 shows the grid for the reference model B (red lines) projected over the grid for LGR (black lines). Note that for the LGR sections, both grid blocks match each other, as intended. The coarse blocks outside the LRG sections are three times larger in the vertical and longer in the horizontal direction over the reference grid. Figure 3 shows the grid for reference model A over the LGR grid scenario. Note that grid corners for reference A are not aligned with the LGR sections or coarse blocks.

\subsection{Description of the Coarse Grid}

The coarse model grid cells measure $100 \times 100 \times 8 \mathrm{~m}$. We consider a region of UNISIM-II-R measuring $2000 \times 2000$ $\times 150 \mathrm{~m}$. Figure 4 shows the base production strategy for flow simulation purposes. The production strategy is inverted-five spot with 1 injector and 4 producers.

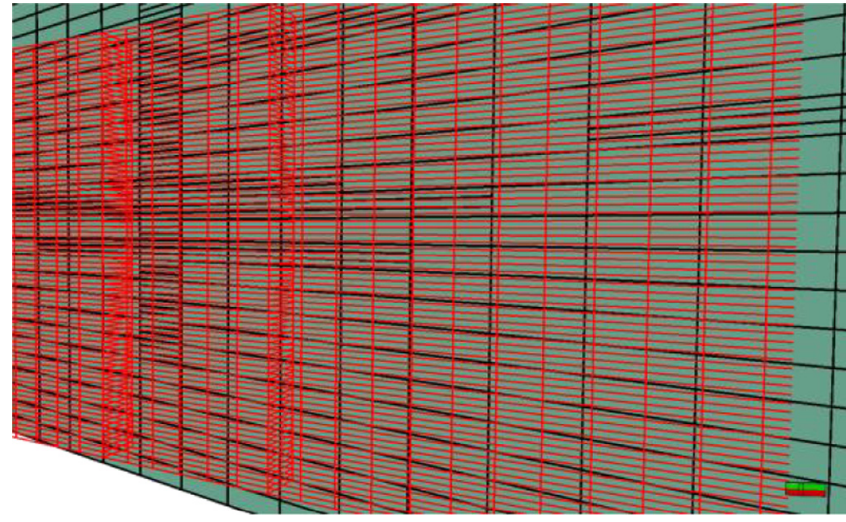

Figure 3

Grid for the reference model A overlapping the grid for LGR scenario.

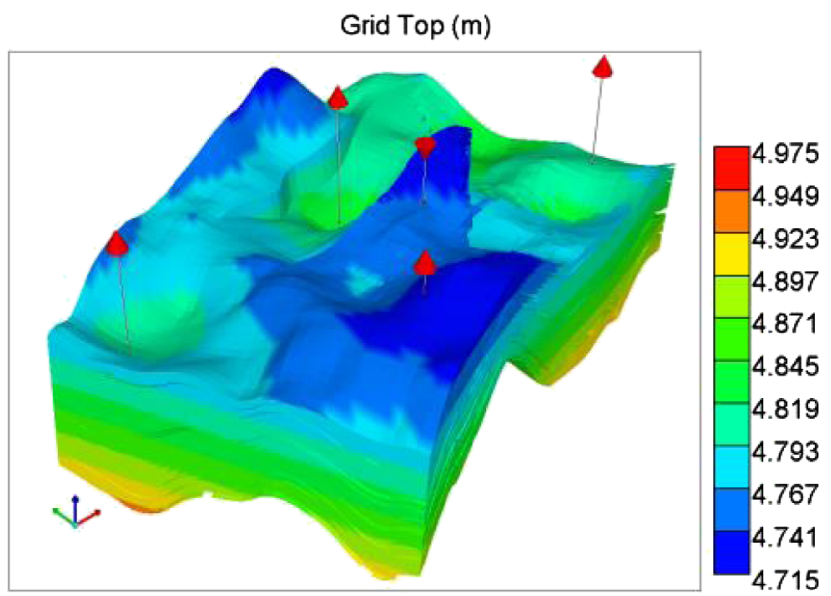

Figure 4

Production strategy for flow simulation purposes.

\subsection{Application of LGR in Conventional Coarse Grid}

Three LGR scenarios are considered: (1) wells, (2) super$\mathrm{k}$, (3) super-k and wells. For all LGR scenarios, the refinement is applied only vertically. For each host cell (coarse block), LGR are defined by three blocks. Figures 5-8 show the sequence of the LGR for well and super-k regions, using permeability as an example. Figure 5 shows the permeability for the coarse model. Figure 6 shows the selected host cells (red blocks) for super-k layers based on a cut-off for permeability. Only cells with permeability above $800 \mathrm{mD}$ are considered for LGR, representing super-k layers. Host cells for wells are defined explicitly in the simulator model. Figure 7 shows the LGR set for host cells. LGR sections and the reference model show good matches (Fig. 8) for LGR sections. These examples are from Case B. 


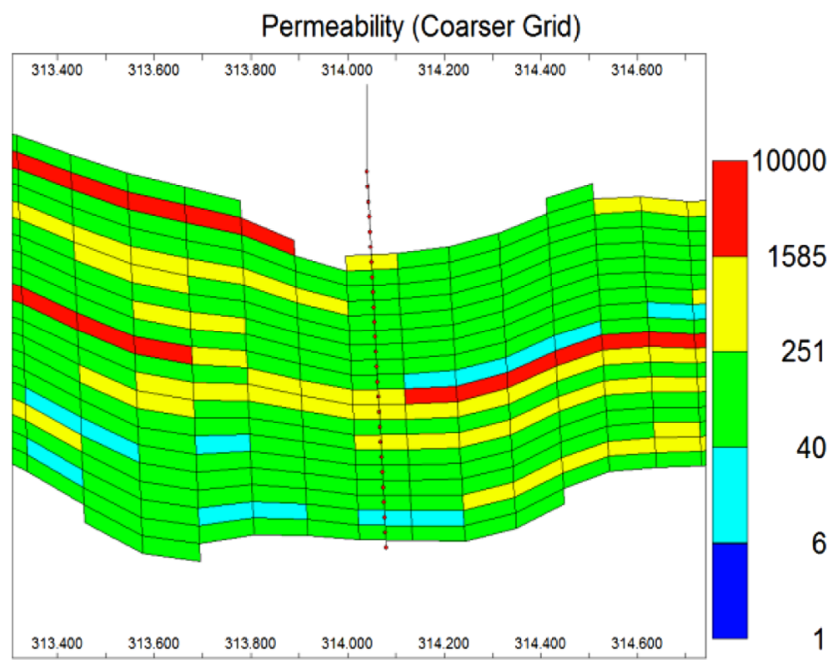

Figure 5

Permeability from coarse grid, used to select host cells.

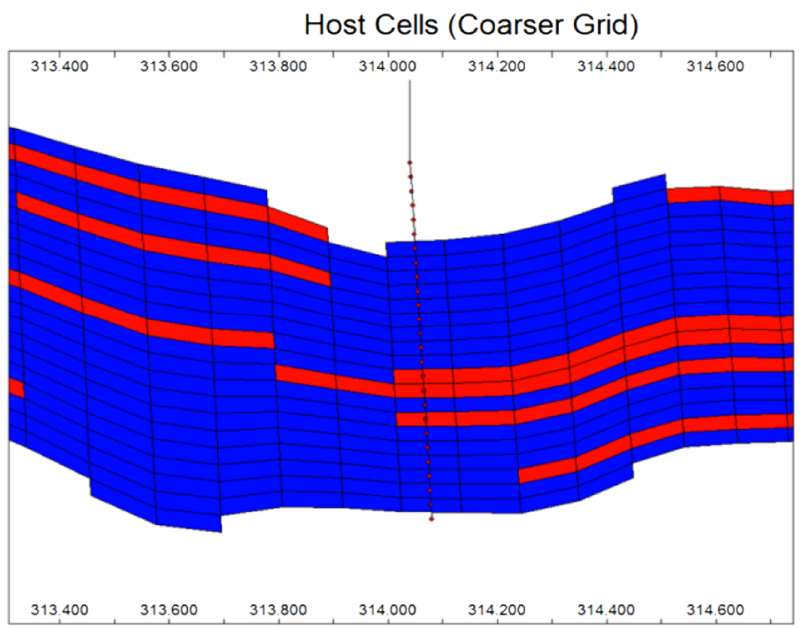

Figure 6

Host cell selection for LGR approach based on a cut-off in permeability.

\subsection{Application of Upscaling Procedure}

The flow-based technique, through the finite difference method, provides the best matching with the reference model for the conventional coarse grid and, therefore, is the best method to compare the benefits of LGR with the conventional coarse grid.

\subsection{Application of LGR SIM}

For the conventional approach, we consider the same LGR scenarios as the previous LGR integrated with upscaling procedures in order to make a proper comparison. Figure 9

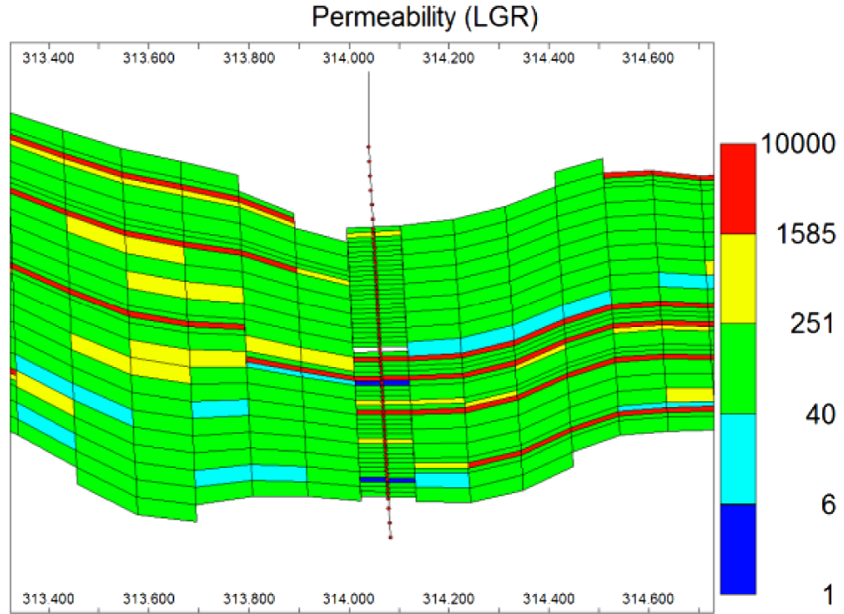

Figure 7

LGR for well and super-k based on host cell location.

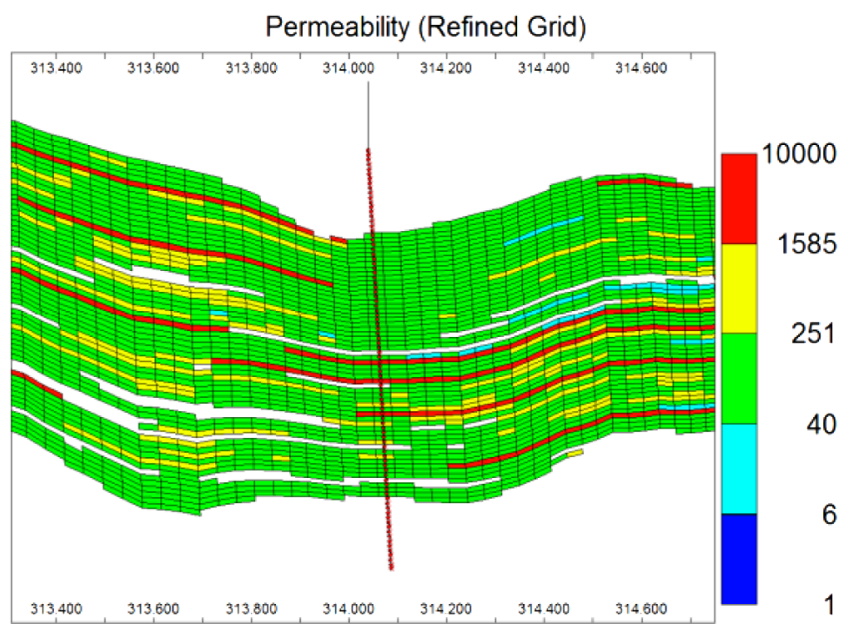

Figure 8

Reference model showing a close match with LGR sections.

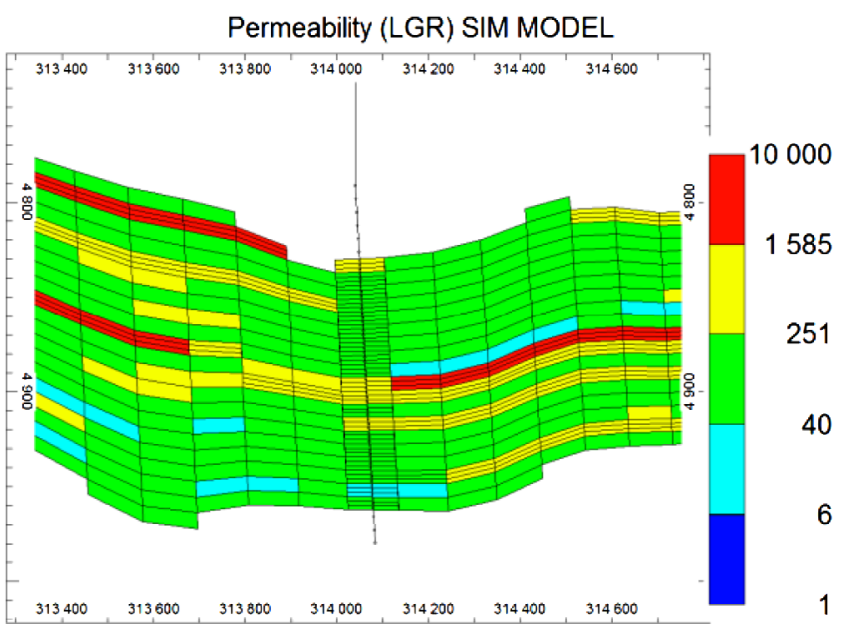

Figure 9

LGR SIM for well and super-k. 
(a)

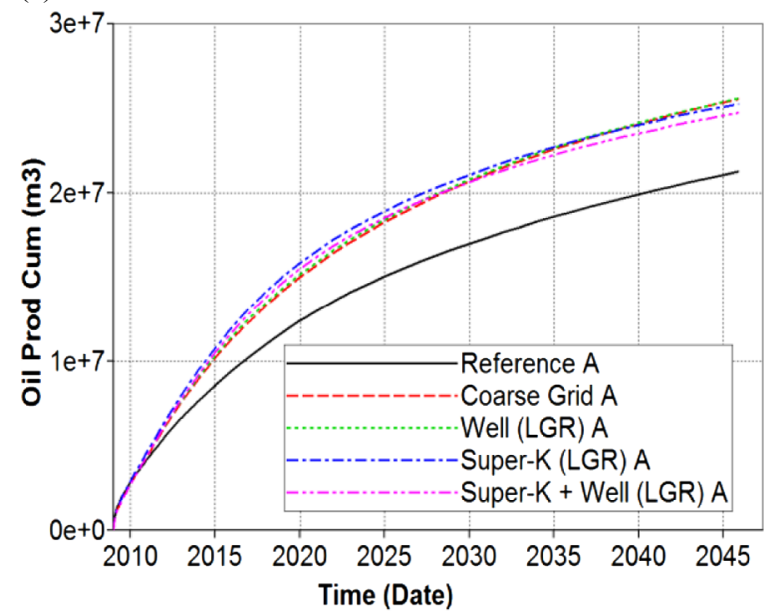

(b)

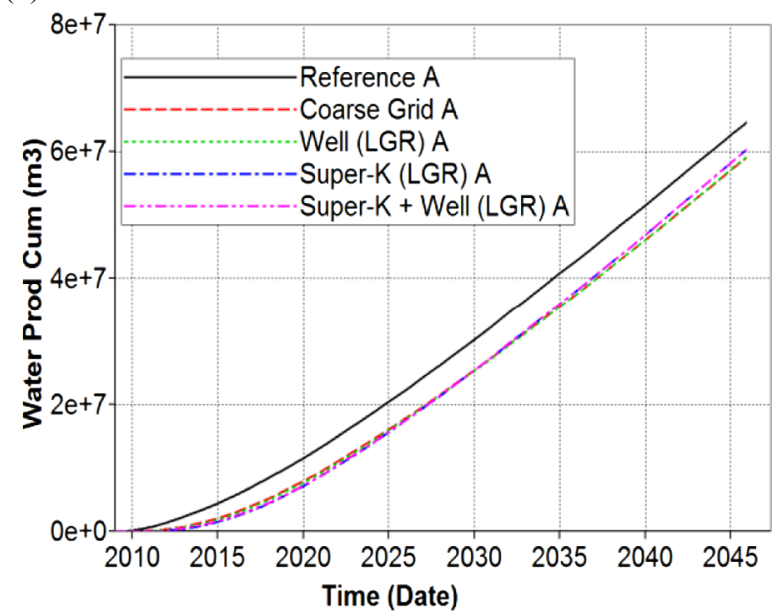

Figure 10

a) Field oil production comparison for the upscaling of reference model A, b) Field water production comparison for the upscaling of reference model A.

(a)

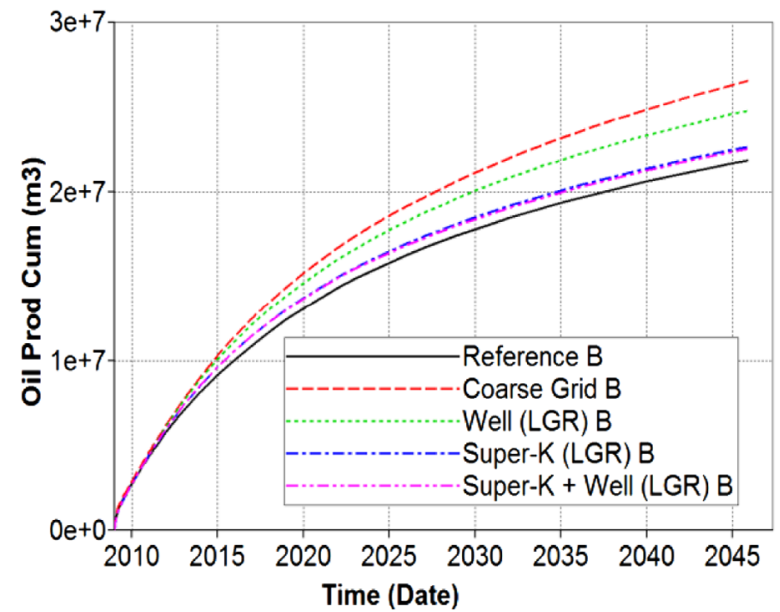

(b)

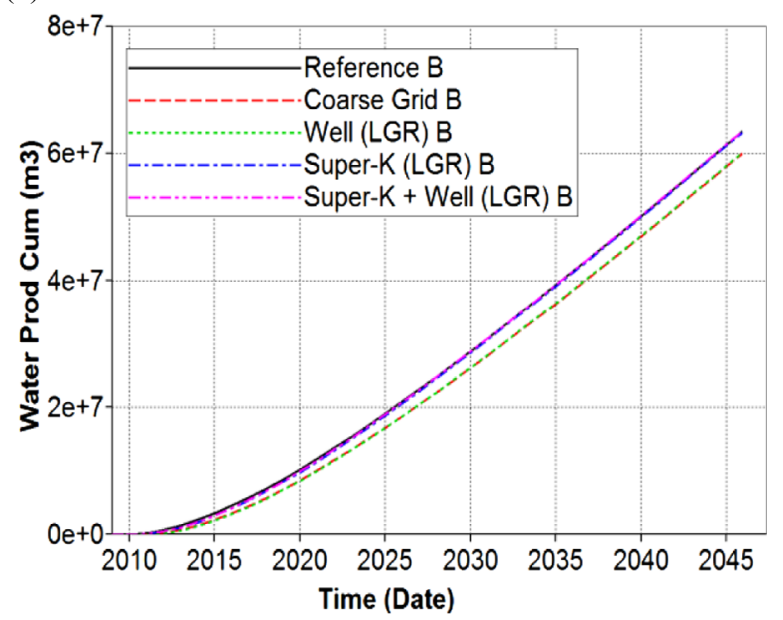

Figure 11

a) Field oil production comparison for the upscaling of reference model B, b) Field water production comparison for the upscaling of reference model B.

shows the LGR set for the host cells, using permeability as example. Note that each refined block has the same value as the host cell.

\section{RESULTS AND DISCUSSION}

The results and discussion section explores the final step of the methodology and shows the dynamic response, based on flow simulation, for all grid scenarios. The single and dualmedium flow models are compared separately.

\subsection{Dynamic Response from Flow Simulation for the Single-Medium Flow Models}

Figures 10 and 11 show the flow simulation results (cumulative oil production and cumulative water production), comparing the upscaled models for reference $\mathrm{A}$ and reference B, respectively. Note that LGR set and conventional coarse grid for the upscaling of reference model A present worse matches than the upscaling for reference model B. Furthermore, LGR showed insignificant improvements comparing 


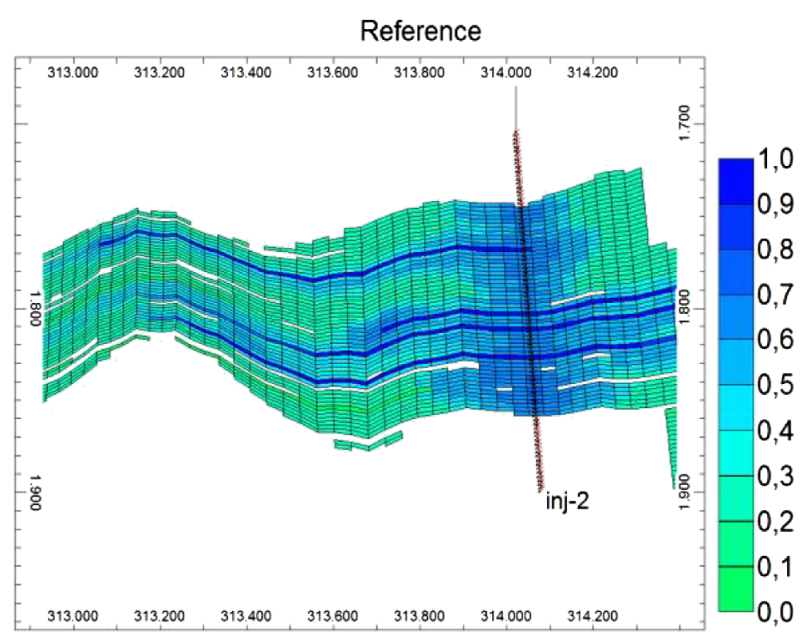

Figure 12

Water front near injector for the reference model.

with conventional coarse grid. The upscaling matching analysis for case B showed an improvement for all simulation grid scenarios (conventional coarse grid and LGR). The use of LGR showed a perfect match with reference solution. These results show that LGR can be inaccurate if grid block corners of the fine-scale geological models and the coarse simulation grid do not match.

The upscaling of refined models with LGR with matching grid block corners (Case B) ensures the same values between each grid despite different averaging methods. However, even with the same bulk volume between refined models and refined blocks from LGR, the mismatch between block corners (Case A) influences the upscaling methods as the equivalent permeability from refined blocks in LGR is underestimated after upscaling. This is particularly critical in super-k regions due to the larger contrasts in permeability. Consequently, the data for LGR blocks are not equal to that of the refined model. Furthermore, we can see a poorer dynamic matching with reference model A, for the coarse grid without LGR compared with case B. This shows that the mismatch between grid block corners affects upscaling and, consequently, the dynamic response.

LGR in the near-well locations avoids the necessity of modifying the well index, in the conventional coarse grid, to calibrate it with the reference solution. However, there are only minor improvements around wells with LGR compared with the conventional coarse grid approach. For super-k with LGR there is a perfect dynamic match with the reference model. The improvement of upscaling matching with LGR in only super-k suggests that the dynamic behavior in the inter-well regions for the thin layers reproduces better results compared with LGR near-wells. This improvement is due to good matching between dynamic data (relative permeability and capillary pressure) and static and dynamic data from the fine-scale model.

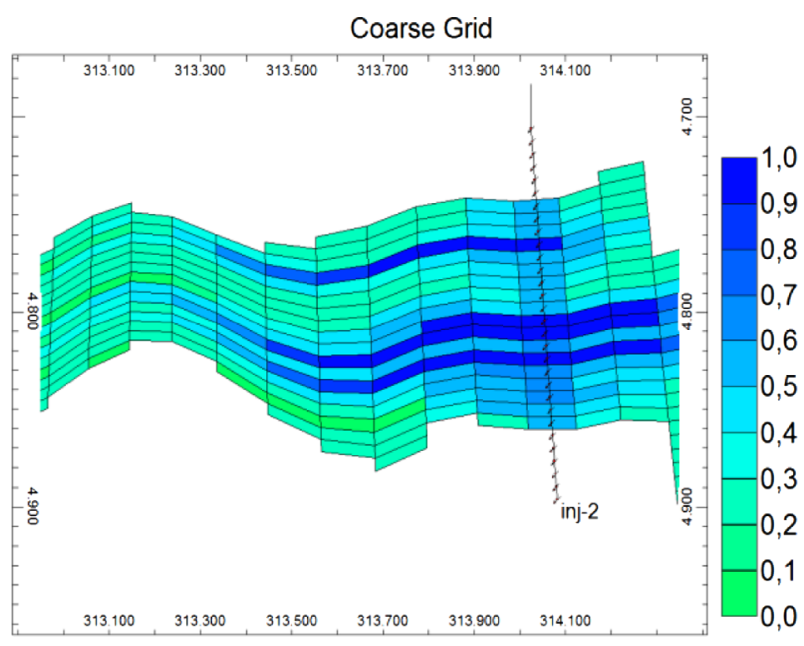

Figure 13

Water front near injector for the conventional coarse grid.

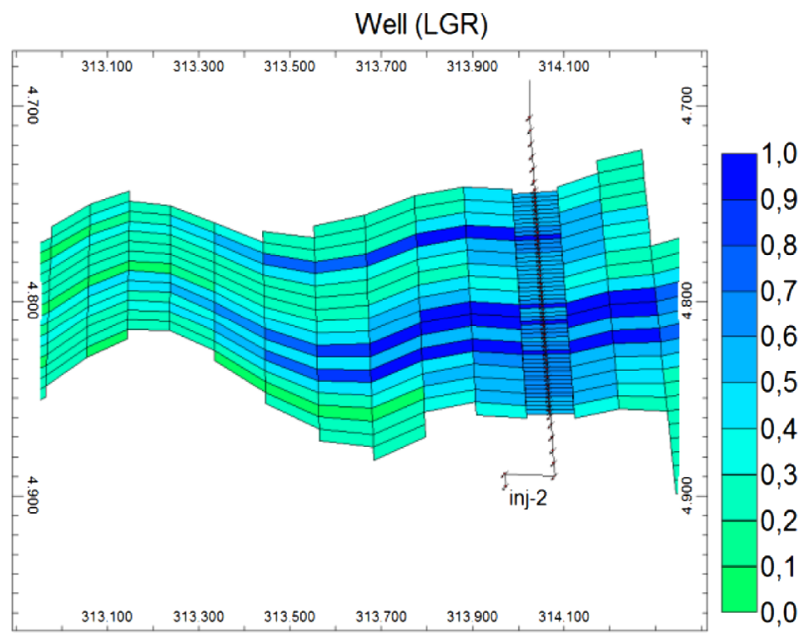

Figure 14

Water front near injector for LGR for wells.

Figure 12 shows the water saturation (for two years of production) near the injector well for the reference model. Figures 13-16 show the water saturation for the conventional coarse grid and with LGR. Note that water saturation shows better inter-well matching with the reference model for grid scenario with LGR in super-k layers (Fig. 14). The enhanced matching is likely due to the closer representation of dynamic data (relative permeability) and through accurate computation of effective permeabilities.

For an enhanced analysis, Figures 17-19 show the rock type property defined in (1) reference model, as (2) LGR in super-k layers, and as (3) host cell, respectively. Note that only the rock type property differs between both scenarios for each refined block in LGR or defined as host cell (one value for the coarse block). The petrophysical distribution is equal for each refined block with LGR. 


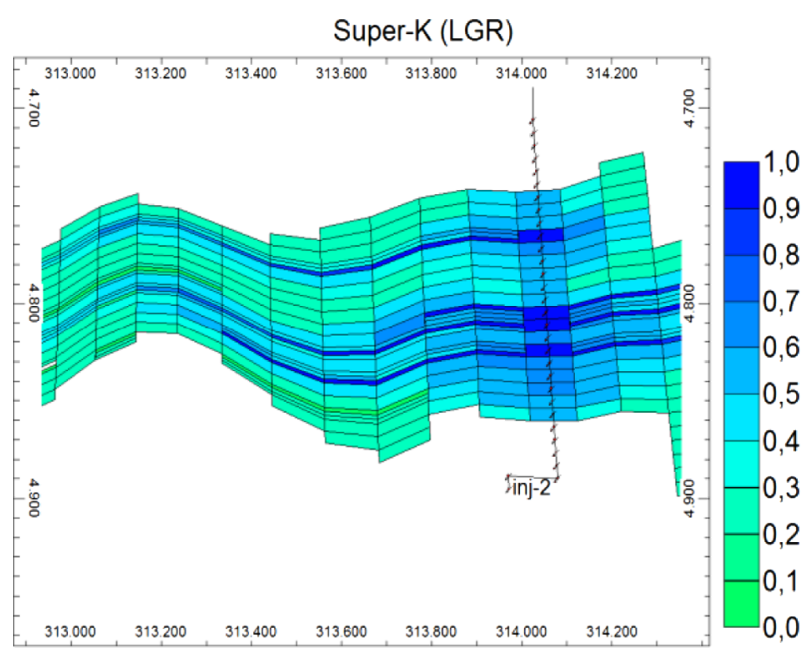

Figure 15

Water front near injector for LGR for super-k.

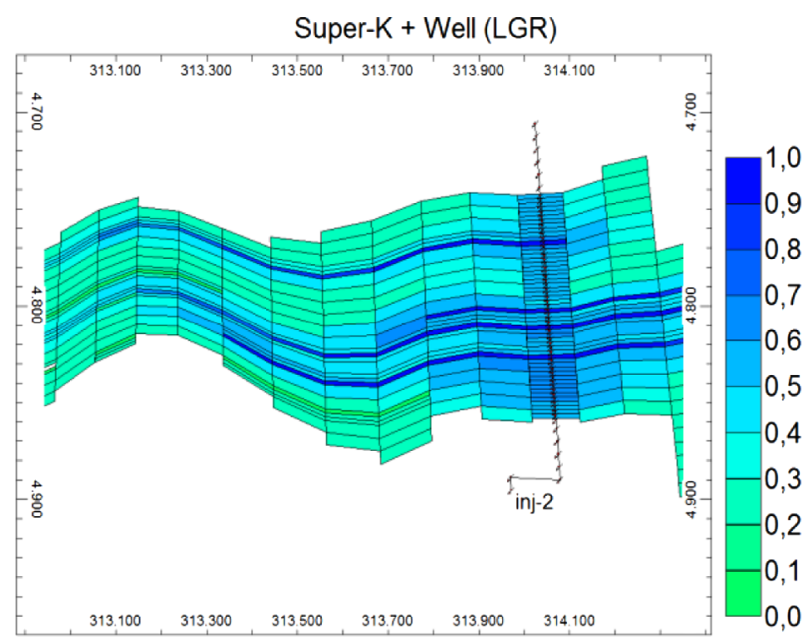

Figure 16

Water front near injector for LGR for super-k and wells.

Figure 20 shows the cumulative oil and water production comparing both scenarios with the reference model. These results show that when relative permeability related to super$\mathrm{k}$ layers is defined in the host cell (coarse block), the grid with LGR presents the worst matches with reference model and closer behavior to conventional coarse model.

Figure 21 shows the water saturation for the case with rock type defined as the host cell. Comparing the water saturation for cases with the rock type defined as host cell and as LGR (Fig. 15), highly saturated blocks are visible near wells. Despite considering LGR for static properties (porosity and effective permeability), the extrapolation of dynamic properties (relative permeability and capillary pressure) to the host cells masks the previous results and produces a response close to the conventional coarse model. This proves that flow-based upscaling techniques can be

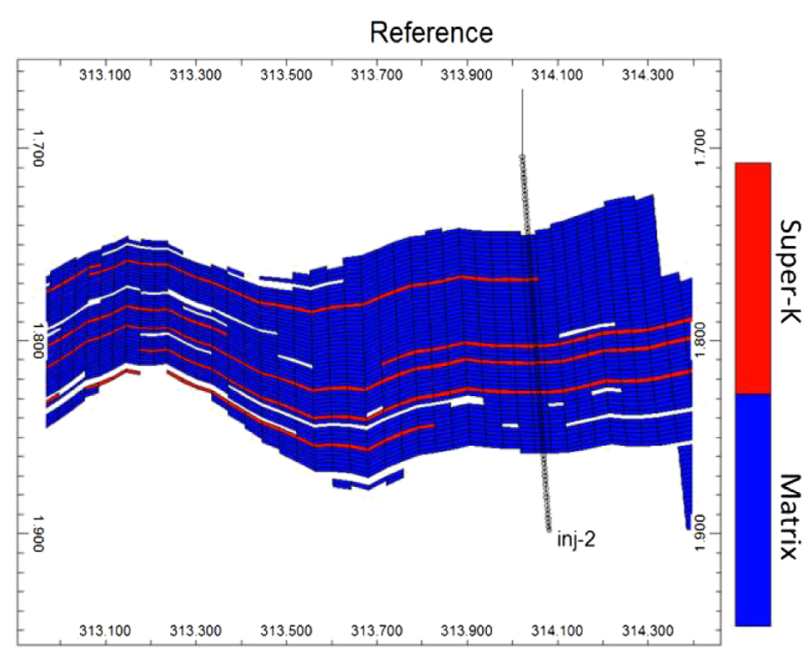

Figure 17

Rock type defined as reference model.

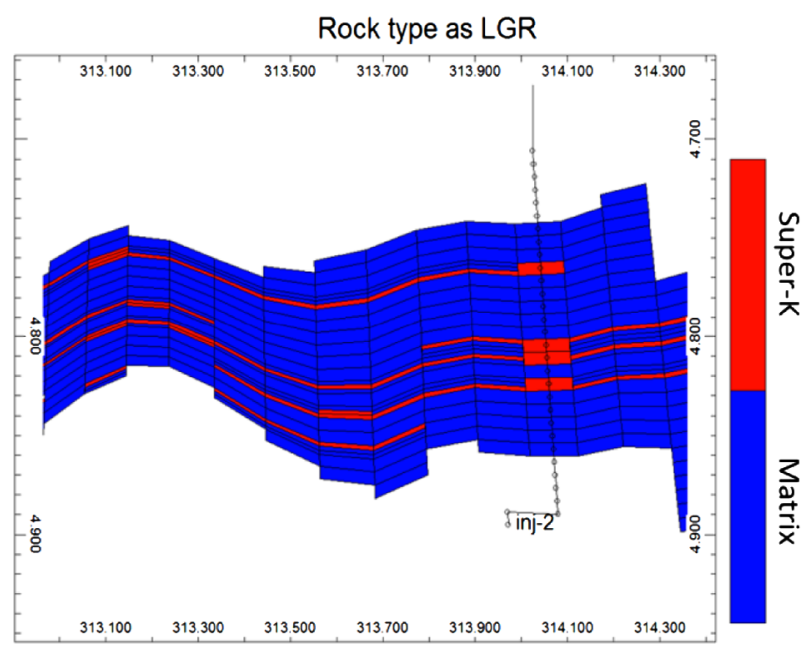

Figure 18

Rock type defined as LGR.

adequate to represent static behavior, but upscaling dynamic data through pseudo-relative permeability curves is essential when using conventional coarse grids. Several works (Hearn, 1971; Al-Otaibi and Al-Majed, 1998; Fayazi et al., 2016; Fahad et al., 2017) present methods to use pseudo-relative permeability curves for upscaling-matching purposes or converting dual-medium flow models into single medium. However, the study of pseudo-relative permeability curves is beyond the scope of this work.

\subsection{Dynamic Response from Flow Simulation for the Dual-Medium Flow Models}

Figure 22 shows the cumulative oil and water production, comparing the upscaling of reference model $\mathrm{B}$ with the inclusion of the fracture system (dual-medium flow model). 


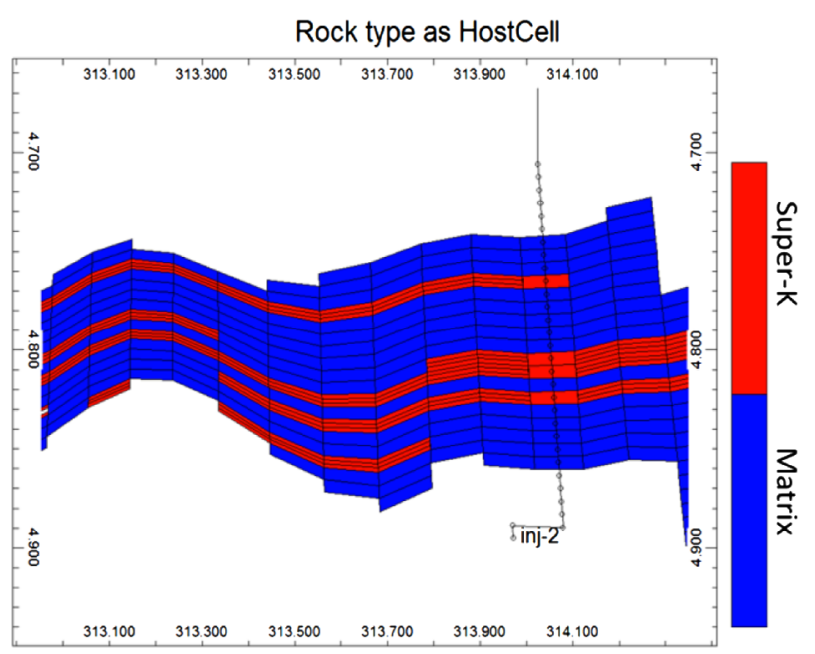

Figure 19

Rock type defined as host cell for LGR.

(a)

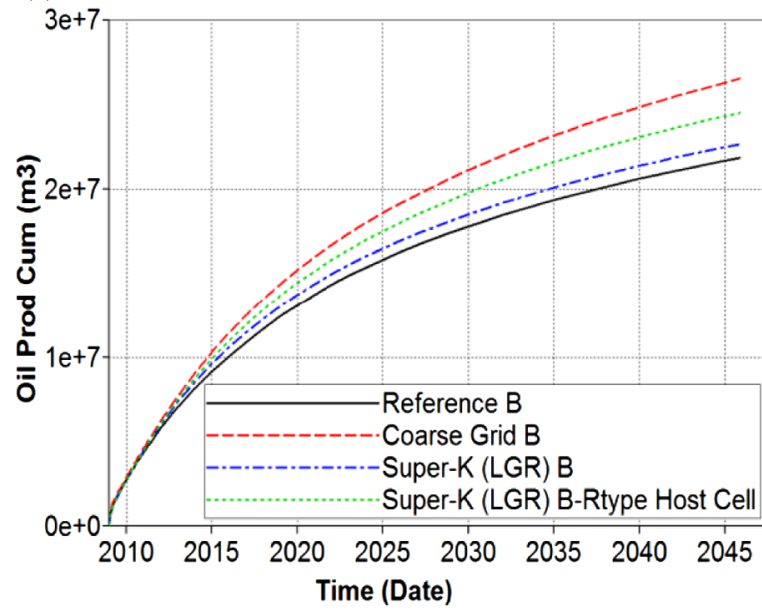

(b)

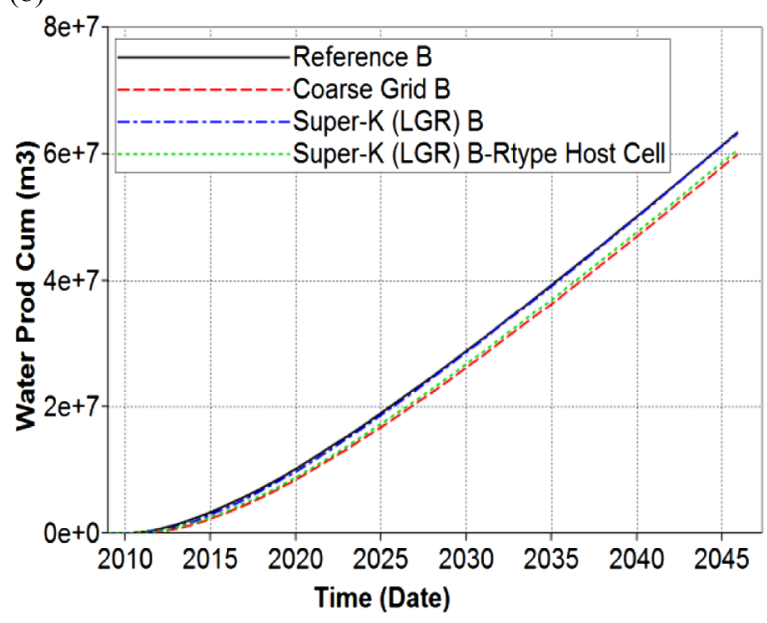

Figure 20

a) Field oil production comparison, b) Field water production comparison.

Note that all coarse grid scenarios present poorer matches compared with the single-medium flow models. The discrete fracture networks are upscaled using an analytical and conventional upscaling approach based on the Oda method (Oda, 1985). As this method is only applicable for wellconnected and uniform fractures, for low density of discrete fractures, the Oda method overestimates the effective block permeability. The UNISIM-II-R is partially fractured and, consequently characterized by a scattered and poorly connected fracture system. The Oda method masks the matching for dual-medium flow model, as the method computes inaccurate effective block permeabilities into the fracture system. Furthermore, given geostatistical software

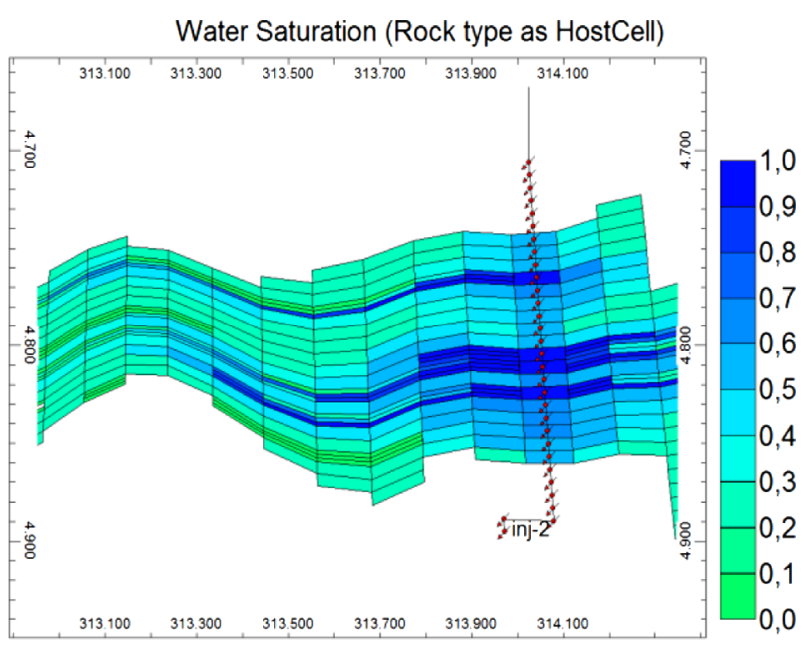

Figure 21

Water saturation for rock type defined as host cell for LGR. limitations, the upscaling of discrete fracture network cannot be applied to LGR.

Figures 23 and 24 show a cross section for matrix and fracture permeability, respectively, with LGR in super-k and well regions for case B. Note that fracture permeability is computed for the host cell; consequently, each block refinement inside the host cell assumes the same value, as opposed to the matrix where permeability is computed for LGR. Therefore, the LGR for dual-medium flow models presents some limitations due to geostatistical software restrictions and Oda method assumptions. For this field, including LGR for super-k shows a small improvement in the dual-medium flow model. 
(a)

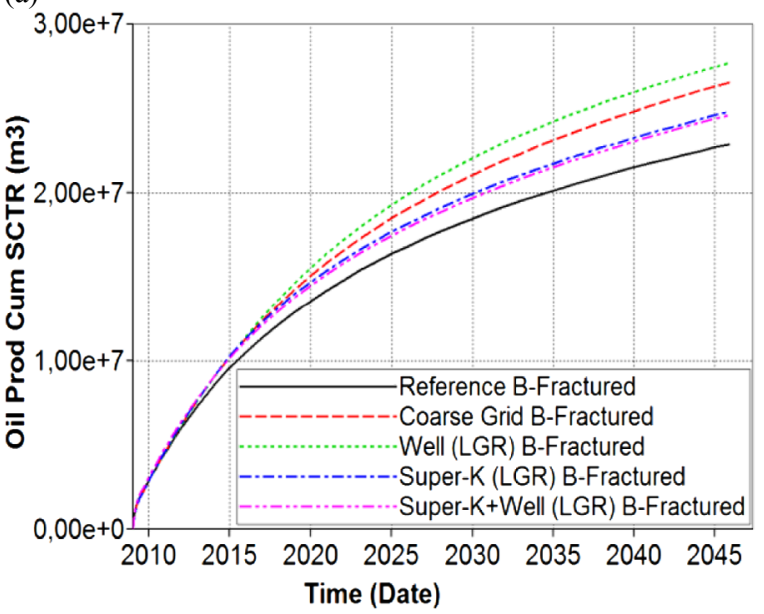

(b)

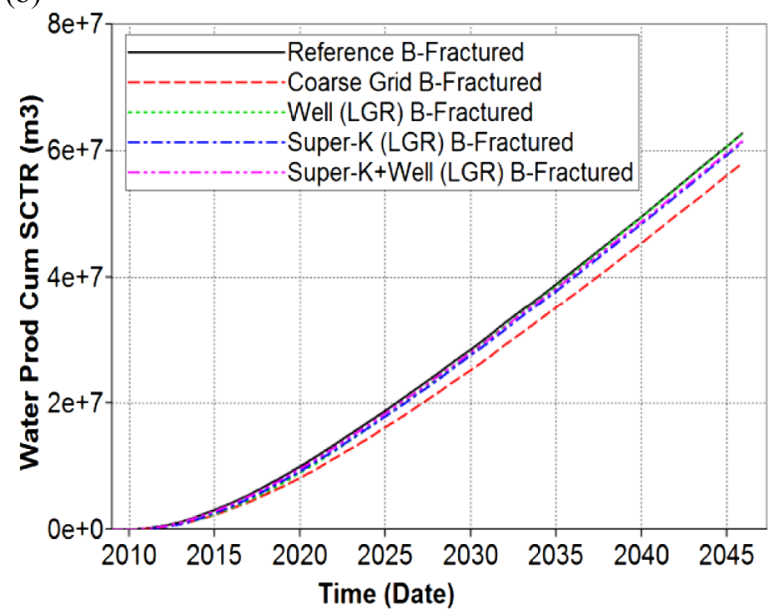

Figure 22

a) Field oil production comparison, b) Field water production comparison.

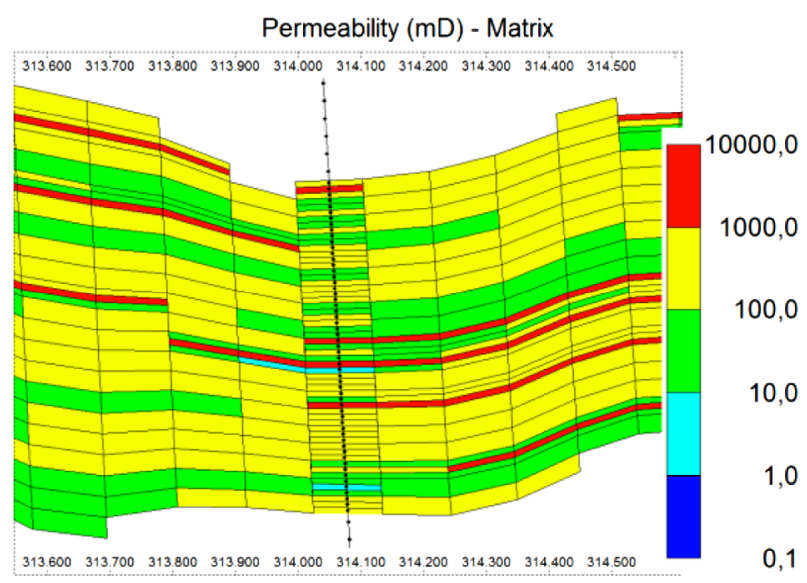

Figure 23

Matrix permeability for LGR.

\subsection{Comparison between LGR Integrated with Upscaling Procedures and the Conventional Approach of LGR SIM}

Figure 25 shows the flow simulation results for the upscaled models comparing LGR integrated with upscaling procedures and LGR SIM (conventional method). This comparison is done considering Case B and the single-medium flow model. Note that LGR SIM presents worse match than LGR integrated with upscaling procedures, especially for cumulative oil production. Comparing with conventional coarse grid, LGR SIM has insignificant improvements. Figure 26 shows the water saturation near injector well for the LGR SIM. Comparing with conventional coarse grid (Fig. 13) it has nearly the same water front and, therefore, a poor match with reference model (Fig. 12), comparing with LGR integrated with upscaling procedures (Fig. 16). These

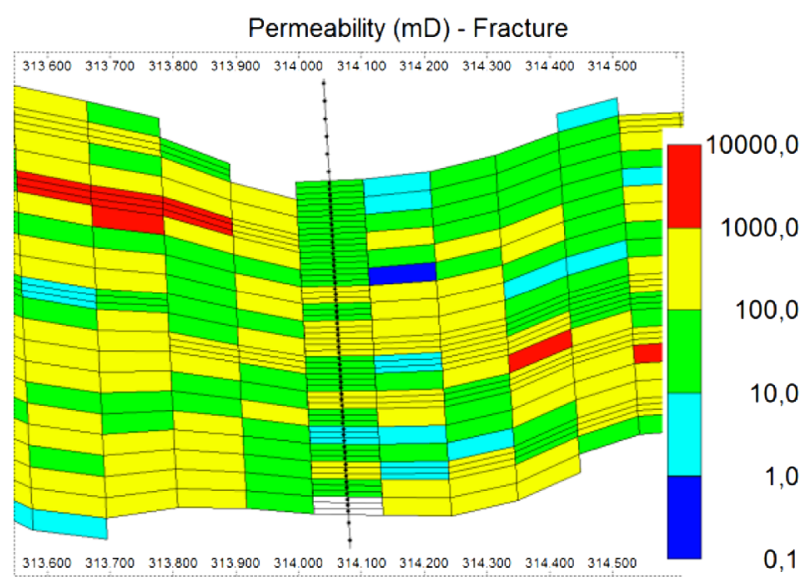

Figure 24

Fracture permeability for LGR.

results show the importance of integrating geological trends in LGR through upscaling procedures. As seen in previous sections, the conventional coarse grid has a poor match with reference model mainly due the extrapolation of relativepermeability from super-k layers for the coarse block. Therefore, as the LGR SIM only replicates the host cell value into the refined blocks, it is expected a small improvement comparing with conventional coarse grid. A small improvement occurs only for LGR in well region as well index calculation is dependent on block size.

\subsection{Quantitative Analysis using NQDS}

We use the Normalized Quadratic Distance with Signal (NQDS) to quantitatively analyze the match, which represents the range of acceptable misfit with respect to the reference solution. NQDS is based on a tolerance applied 
(a)

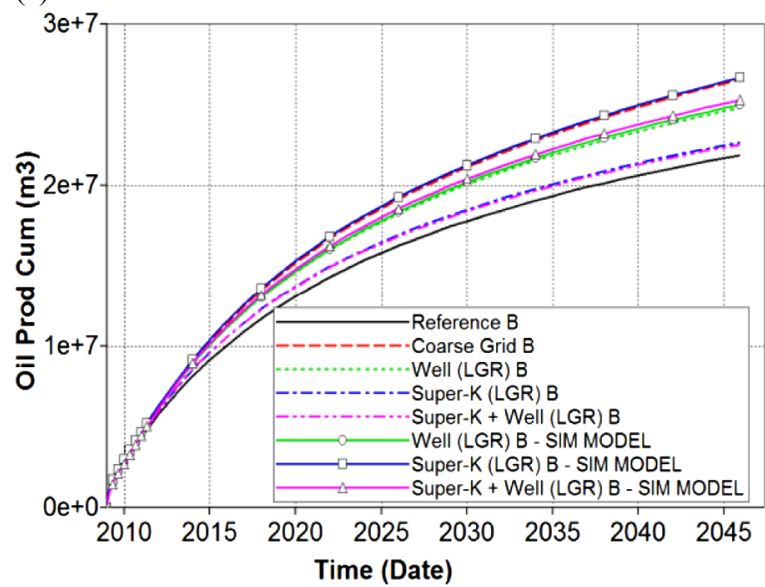

(b)

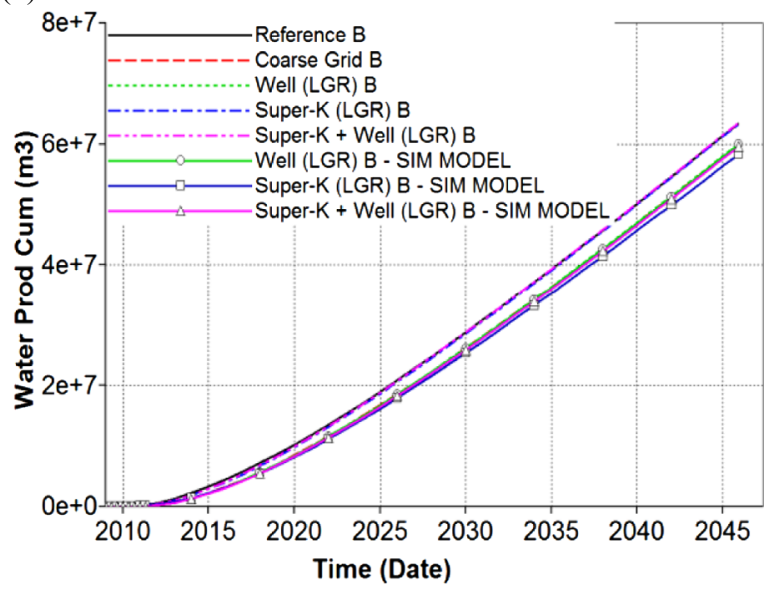

Figure 25

a) Field oil production comparison, b) Field water production comparison.

Super-K + Well (LGR) - SIM MODEL

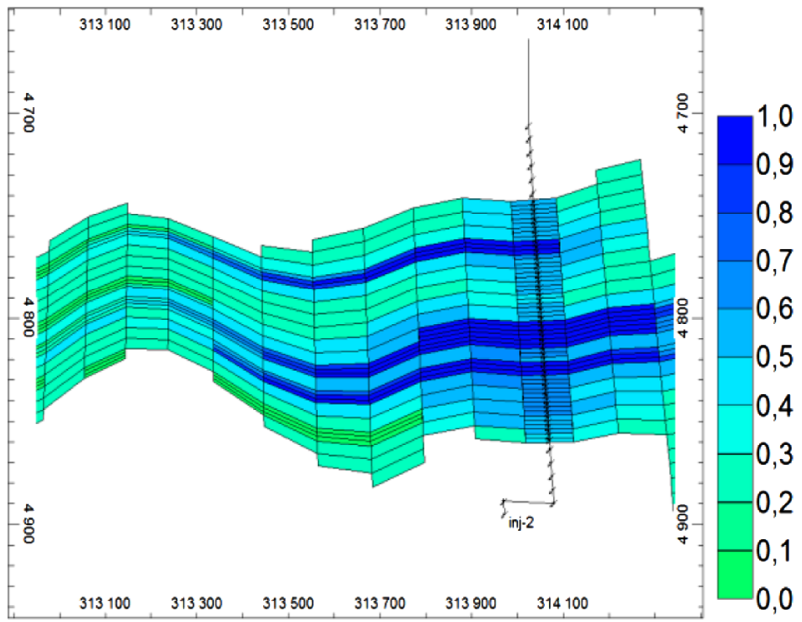

Figure 26

Water saturation for LGR SIM.

to the reference solution generating a confidence interval according to the tolerance. Negative NQDS means that a given curve related to coarse solution is (predominantly) below the reference and vice-versa. Details regarding the NQDS computation are found in Maschio and Schiozer (2016). Figures 27-30 show the NQDS plot for each case. A tolerance of $10 \%$ was applied for both cumulative oil $(\mathrm{Np})$ and cumulative water production $(\mathrm{Wp})$.

The NQDS is plotted for each producer well and for the field. Each plot has a full view (top side) and a zoom view (down side) to highlight the misfit with the reference solution. Figure 27 shows that, except for NP (P2), all models from case $\mathrm{A}$ are outside tolerance range $[-1,+1]$. Figure 28 shows that models with LGR in super-k regions are within the tolerance for nearly all data. The full views
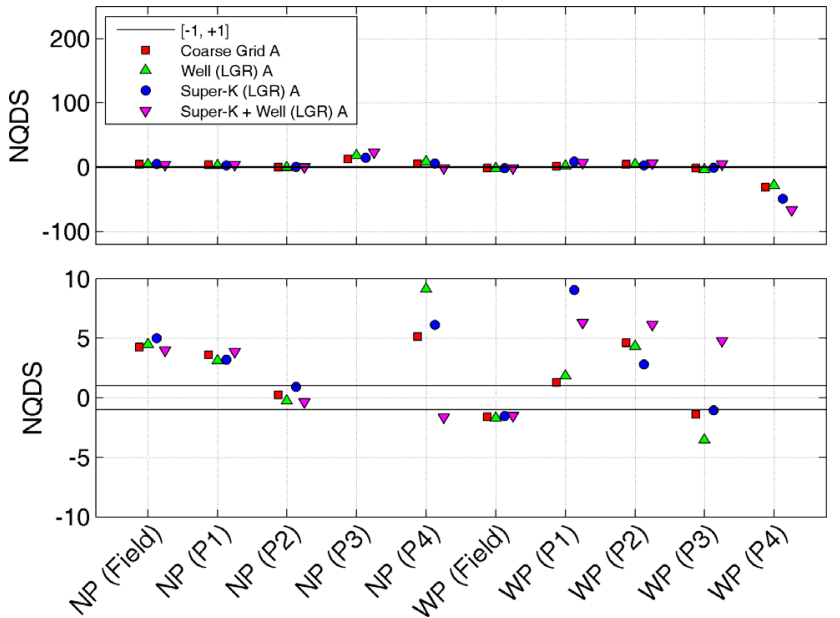

Figure 27

NQDS for Case A.

show that the coarse grid is outside the tolerance, particularly for cumulative water production. The zoom view shows that LGR in near-well regions also produces a poor match compared with LGR in super-k regions. Figure 29 shows the NQDS plot for the dual-medium case. Comparing with NQDS for case B (single-medium), for the zoom view, we can see that all models are more dispersed from the reference for all attributes with the exception of cumulative oil production for producer 3 and cumulative water production for the field. The model with LGR in nearwell regions shows poorer results than for the coarse grid for some wells and cumulative oil production for the field.

The addition of a poorly connected fractured system masks the advantages of LGR, especially in near-well regions as the upscaling method for discrete fracture networks leads to over-estimated permeabilities. Well 

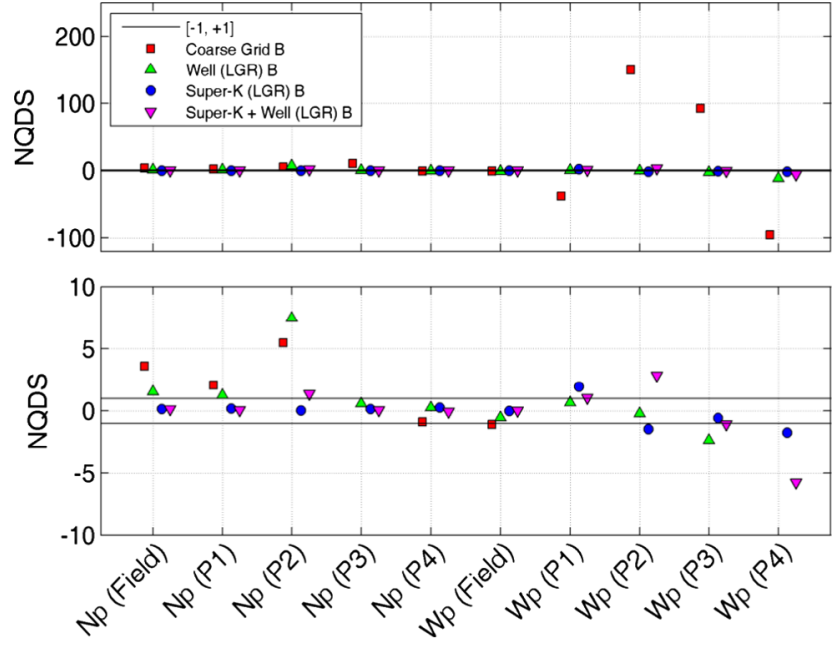

Figure 28

NQDS for Case B.
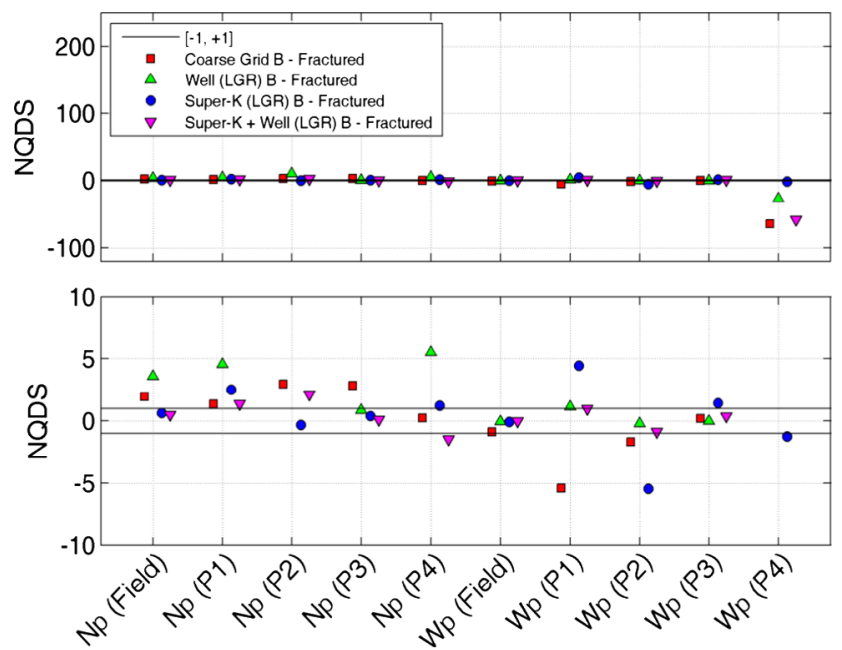

Figure 29

NQDS for Case B (dual-medium flow model).

productivity for dual-medium flow models is dependent on well indexes for matrix and fracture systems. As the Oda upscaling method over-estimates the permeability for poorly connected fracture systems, we expect a weak estimation of well productivity for the fracture system. Furthermore, due to the limitation of commercial geostatistical software, discrete fracture networks cannot be upscaled for LGR. So, effective permeabilities for fracture system are computed for the host cell (coarse block).

These results agree with the previous qualitative analysis for single and dual-medium flow models.

Figure 30 shows the NQDS plot comparing the LGR SIM and the proposed LGR integrated with upscaling procedures. We can see that all LGR SIM are outside the tolerance range for cumulative oil production and more dispersed from the reference for cumulative water production comparing to
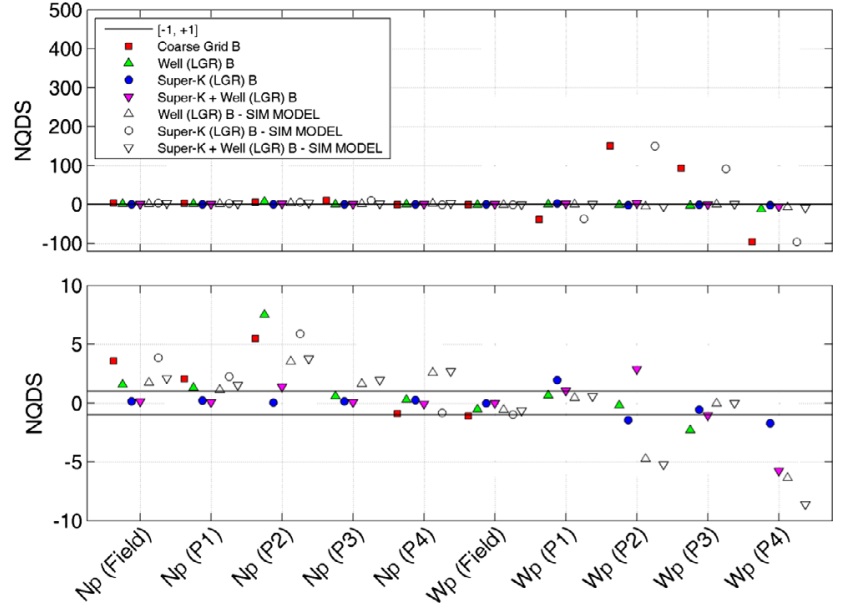

Figure 30

NQDS comparing the conventional LGR in simulation model and the proposed LGR linked with upscaling procedures.

Table 1

Simulation time consumption.

\begin{tabular}{|c|c|c|c|}
\hline Case & \multicolumn{2}{|c|}{ Single/Dual medium } & $\begin{array}{l}\text { Simulation time } \\
\text { consumption ( } \mathrm{sec})\end{array}$ \\
\hline Reference A & \multicolumn{2}{|l|}{ Single } & 17 \\
\hline \multirow{2}{*}{ Reference B } & \multicolumn{2}{|l|}{ Single } & 1047 \\
\hline & \multicolumn{2}{|l|}{ Dual } & 193885 \\
\hline \multirow{2}{*}{ Coarse Grid } & \multicolumn{2}{|l|}{ Single } & 9 \\
\hline & \multicolumn{2}{|l|}{ Dual } & 148 \\
\hline \multirow{6}{*}{ LGR } & \multirow{2}{*}{ Well } & Single & 16 \\
\hline & & Dual & 280 \\
\hline & \multirow{2}{*}{ Super-K } & Single & 23 \\
\hline & & Dual & 693 \\
\hline & \multirow{2}{*}{ Super-k + Well } & Single & 38 \\
\hline & & Dual & 992 \\
\hline \multirow{3}{*}{ LGR (SIM MODEL) } & Well & \multirow{3}{*}{ Single } & 17 \\
\hline & Super-K & & 25 \\
\hline & Super-k + Well & & 41 \\
\hline
\end{tabular}

LGR integrated with upscaling procedures. These results agree with the previous qualitative analysis comparing the proposed and the conventional methods.

\subsection{Simulation Time Consumption}

Table 1 shows the simulation time consumption for all cases. We did not present time consumption for reference model A for the dual-medium flow model as the single medium 
upscaling for the corresponding cases already presented a poor match with reference model. Note that there is an increase in time consumption for the dual-medium flow models and an increased number of refined blocks (LGR). Based on the previous results, LGR only on super-k layers is the best grid scenario as the match with reference model provided the same results as LGR in super-k and wells but with minimal time consumption. The higher time consumption and similar results (when compared to the conventional coarse grid) suggest limited usefulness for dual-medium flow models with LGR. For LGR SIM the time consumption is almost equal to LGR integrated with upscaling procedures.

\section{CONCLUSION}

This work proposes LGR integrated with upscaling procedures to better represent laminated reservoirs in flow simulation. This study was performed in a complex geological field characterized by highly permeable thin layers and fractures. The comparison between conventional coarse grid and LGR integrated with upscaling procedures showed that:

- Flow simulation with LGR may inaccurately represent the high fidelity model (fine-scale geological model) if grid block corners do not match between both grids;

- Flow simulation with LGR in the inter-well regions for the super-k layers provided better matches with the fine-scale geological model compared with LGR in near-wells locations and conventional coarse grids;

- The extrapolation of relative-permeability data from LGR in super-k layers for the host cell (coarse block) led to similar behavior to the conventional coarse model and, consequently, a poorer match with the reference model;

- Flow-based upscaling techniques can adequately represent the static behavior for coarse grids while upscaling dynamic data using pseudo-relative permeability curves can improve the upscaling matching for conventional coarse grids;

- LGR in dual-medium flow models presented several limitations. Commercial geostatistical software does not upscale DFN for the refined blocks in LGR sections (only host cells), and the analytical upscaling method (Oda, 1985) is only applicable for highly connected fractured networks. Therefore, including LGR for super-k area showed minimal improvements in dual-medium flow models.

The comparison between LGR integrated with upscaling procedures and the conventional LGR approach integrated with simulation model showed that:

- LGR in simulation model replicates the host cell value into the refined blocks and, therefore, as observed in the conventional coarse grid, the relative-permeability from super-k layers is extrapolated for the coarse block, which leads to a poorer match with the reference model.
- LGR integrated with simulation model lack to represent the vertical anisotropy from super-k trends, as the conventional coarse grid, and, therefore, appears to be unsuitable for laminated reservoirs.

To accurately represent highly permeable thin layers in flow simulations, we note the following important steps:

- Pseudo-relative permeability curves should be considered for conventional coarse grids or LGR scenarios for dualmedium flow models;

- The assignment of rock-fluid data for refined blocks in LGR sections through a rock-type property is essential for accuracy when performing LGR;

- Flow simulation through LGR near-wells is not recommended due to increased simulation time and minimal improvements in flow response compared with the fine grid model;

- The upscaling of geological models for the coarse grid with LGR in super-k regions provided a close dynamic representation with geological characterization.

This work shows the importance of integrating geological trends in LGR through upscaling procedures and presents an analysis that can be useful to integrate highly permeable thin layers into flow simulators and prevent problems related to upscaling procedures that mask geological characterization.

\section{ACKNOWLEDGMENTS}

The authors are grateful to the Center of Petroleum Studies (Cepetro-Unicamp/Brazil), PETROBRAS S/A, UNISIM, ANP and the Petroleum Engineering Department (DEPFEM-Unicamp/Brazil) for their support of this work. The authors are also grateful to Schlumberger Information Solution for the use of Petrel ${ }^{\circledR}$.

\section{REFERENCES}

Ahr W.M. (2008) Geology of carbonate reservoirs: the identification, description, and characterization of hydrocarbon reservoirs in carbonate rocks, John Wiley and Sons, New Jersey.

Al-Dhafeeri A.M., Nasr-El-Din H.A. (2007) Characteristics of high-permeability zones using core analysis, and production logging data, J. Pet. Sci. Eng. 55, 1-2, 18-36, http://dx.doi.org/ 10.1016/j.petrol.2006.04.019.

Al-Otaibi S.S., Al-Majed A. (1998) Factors affecting pseudo relative permeability curves, J. Pet. Sci. Eng. 21, 3, 249-261, http://dx.doi.org/10.1016/S0920-4105(98)00070-9.

Boyd A., Souza A., Carneiro G., Machado V., Trevisan W., Santos B., Netto P., Bagueira R., Polinski R., Bertolini A. (2015) Presalt Carbonate Evaluation for Santos Basin, Offshore Brazil, Soc. Petrophys. Well-log Analyst. 56, 6, 577-591.

Corbett P.W.M., Jensen J.L. (1992) Estimating the mean permeability: how many measurements do you need? First Break 10, 3, 89-94. 
Correia M.G., Maschio C., Schiozer D.J., Santos M.S. (2014) Upscaling approach for meso-scale heterogeneities in naturally fractured carbonate reservoirs, J. Pet. Sci. Eng. 115, 90-101, http://doi.org/10.1016/j.petrol.2014.01.008.

Correia M., Hohendorff J., Gaspar A.T.F.S., Schiozer D. (2015) UNISIM-II-D: Benchmark case proposal based on a carbonate reservoir, in: SPE Latin American and Caribbean Petroleum Engineering Conference, Quito, Ecuador, http://dx.doi.org/ 10.2118/177140-MS.

Ding D.Y. (2004) Near-well upscaling for reservoir simulations, Oil Gas Sci. Technol. 59, 2, 157-165.

Ding D.Y. (2011) Coupled simulation of near-wellbore and reservoir models, J. Pet. Sci. Eng. 76, 21-36.

Ding D.Y., Wu Y.S., Jeannin L. (2014) Efficient simulation of hydraulic fractured wells in unconventional reservoirs, J. Pet. Sci. Eng. 122, 631-642.

Eltom H., Makkawi M., Abdullatif O., Alramadan K. (2013) Highresolution facies and porosity models of the upper Jurassic Arab-D carbonate reservoir using an outcrop analogue, central Saudi Arabia, Arab. J. Geosci. 6, 11, 4323-4335.

Fahad M., Hussain F., Rahman S.S., Cinar Y. (2017) Experimental investigation of upscaling relative permeability for two-phase flow in fractured porous media, J. Pet. Sci. Eng. 149, 367-382, http://dx.doi.org/10.1016/j.petrol.2016.10.013.

Fayazi A., Bagherzadeh H., Shahrabadi A. (2016) Estimation of pseudo relative permeability curves for a heterogeneous reservoir with a new automatic history-matching algorithm, J. Pet. Sci. Eng. 140, 154-163, http://dx.doi.org/10.1016/j. petrol.2016.01.013.

Garland J., Neilson J., Laubach S.E., Whidden K.J. (2012) Advances in carbonate exploration and reservoir analysis, Geological Society, London, Special publications, 370, pp. 1-15.

Hearn C.L. (1971) Simulation of stratified waterflooding by pseudo relative permeability curves, J. Pet. Technol. 23, 7, 805-813, http://dx.doi.org/10.2118/2929-PA.

Kheriji W., Masson R., Moncorge A. (2015) Nearwell local space and time refinement in reservoir simulation, Math. Comp. Simul. 118, 273-292, http://dx.doi.org/10.1016/j.mat com.2014.11.022.
Li H., Chen Y., Rojas D., Kumar M. (2014) Development and application of near-well multiphase upscaling for forecasting of heavy oil primary production, J. Pet. Sci. Eng. 113, 81-93, http://dx.doi.org/10.1016/j.petrol.2014.01.002.

Maschio C., Schiozer D.J. (2016) Probabilistic history matching using discrete latin hypercube sampling and nonparametric density estimation, J. Pet. Sci. Eng. 147, 98-115, https://doi. org/10.1016/j.petrol.2016.05.011.

Noetinger B., Zargar G. (2004) Multiscale description and upscaling of fluid flow in subsurface reservoirs. Oil Gas Sci. Technol. 59, 2, 119-139, https://doi.org/10.2516/ogst:2004010.

Oda M. (1985) Permeability tensor for discontinuous rock mass, Geotechnique 35, 4, 483-495.

Panja P., Conner T., Deo M. (2013) Grid sensitivity studies in hydraulically fractured low permeability reservoirs, J. Pet. Sci. Eng. 112, 78-87, http://dx.doi.org/10.1016/j.pet rol.2013.10.009.

Peng X., Liang B., Du Z., Wang M. (2017) Practical simulation of multi-porosity reservoirs through existing reservoir simulator, J. Pet. Sci. Eng. 151, 409-420, http://dx.doi.org/10.1016/j. petrol.2017.01.027.

Preux C., Ravalec M., Enchéry G. (2016) Selecting an appropriate upscaled reservoir model based on connectivity analysis, Oil Gas Sci. Technol. 71, 60, https://doi.org/10.2516/ogst/ 2016015.

Voelker J., Liu J., Caers J. (2003) A geostatistical method for characterizing superpermeability from flow-meter data: application to Ghawar field, in: SPE Annual Technical Conference and Exhibition held in Denver, Colorado, USA, October 5-8, http://dx.doi.org/10.2118/84279-MS.

Manuscript submitted in 24 August 2017 Manuscript accepted in 15 December 2017 Published online in February 2018 